\title{
Abnormal composition of gut microbiota is associated with resilience versus susceptibility to inescapable electric stress
}

\author{
Kai Zhang ${ }^{1,2}$, Yuko Fujita', Lijia Chang ${ }^{1}$, Youge Qu', Yaoyu Pu' ${ }^{1}$, Siming Wang ${ }^{1}$, Yukihiko Shirayama ${ }^{1,3}$ and
} Kenji Hashimoto (iD ${ }^{1}$

\begin{abstract}
Increasing evidence indicates that abnormalities in the composition of gut microbiota might play a role in stress-related disorders. In the learned helplessness $(\mathrm{LH})$ paradigm, $\sim 60-70 \%$ rats are susceptible to $\mathrm{LH}$ in the face of inescapable electric stress. The role of gut microbiota in susceptibility in the LH paradigm is unknown. In this study, male rats were exposed to inescapable electric stress under the LH paradigm. The compositions of gut microbiota and short-chain fatty acids were assessed in fecal samples from control rats, non-LH (resilient) rats, and LH (susceptible) rats. Members of the order Lactobacillales were present at significantly higher levels in the susceptible rats than in control and resilient rats. At the family level, the number of Lactobacillaceae in the susceptible rats was significantly higher than in control and resilient rats. At the genus level, the numbers of Lactobacillus, Clostridium cluster III, and Anaerofustis in susceptible rats were significantly higher than in control and resilient rats. Levels of acetic acid and propionic acid in the feces of susceptible rats were lower than in those of control and resilient rats; however, the levels of lactic acid in the susceptible rats were higher than those of control and resilient rats. There was a positive correlation between lactic acid and Lactobacillus levels among these three groups. These findings suggest that abnormal composition of the gut microbiota, including organisms such as Lactobacillus, contributes to susceptibility versus resilience to LH in rats subjected to inescapable electric foot shock. Therefore, it appears likely that brain-gut axis plays a role in stress susceptibility in the LH paradigm.
\end{abstract}

\section{Introduction}

Resilience is adaptation in the face of stress and adversity. It is well known that humans display wide variability in their responses to stress. Increasing amounts of evidence show that resilience might be mediated by adaptive changes in several neural circuits, including numerous molecular and cellular pathways ${ }^{1-11}$. An understanding of the molecular and cellular mechanisms underlying resilience will facilitate the discovery of new therapeutic drugs for stress-related psychiatric disorders,

Correspondence: Kenji Hashimoto (hashimoto@faculty.chiba-u.jp)

'Division of Clinical Neuroscience, Chiba University Center for Forensic Mental Health, Chiba 260-8670, Japan

2Department of Psychiatry, Chaohu Hospital of Anhui Medical University, 238000 Hefei, China

Full list of author information is available at the end of the article. but the detailed mechanisms underlying resilience and susceptibility remain unclear.

The brain-gut-microbiome axis is a complex, bidirectional signaling system between the brain and the gut microbiota $^{12-16}$. Accumulating studies suggest that an abnormal composition of the gut microbiota contributes to the pathophysiology of depression ${ }^{17-21}$ and the antidepressant effects of certain potential compounds ${ }^{22-29}$. Previously, we reported that the presence of Bifidobacterium in the gut microbiome confers stress resilience in a chronic social defeat stress (CSDS) model $^{30}$. It has been shown that $\sim 30-40 \%$ of rats are resilient to inescapable electric stress in the learned helplessness (LH) paradigm $^{31-34}$; however, the role of gut microbiota in the production of this resilience has not yet been investigated. Microbes in the gut can produce short-chain fatty acids.

\section{(c) The Author(s) 2019}

(c) (i) Open Access This article is licensed under a Creative Commons Attribution 4.0 International License, which permits use, sharing, adaptation, distribution and reproduction c. in any medium or format, as long as you give appropriate credit to the original author(s) and the source, provide a link to the Creative Commons license, and indicate if changes were made. The images or other third party material in this article are included in the article's Creative Commons license, unless indicated otherwise in a credit line to the material. If material is not included in the article's Creative Commons license and your intended use is not permitted by statutory regulation or exceeds the permitted use, you will need to obtain permission directly from the copyright holder. To view a copy of this license, visit http://creativecommons.org/licenses/by/4.0/. 
The presence and abundance of such acids could possibly be used as an indicator of the types of bacteria present in the gut. However, it is also currently unknown how the altered composition of the gut microbiota affects the concentration of short-chain fatty acids in fecal samples.

The purpose of this study was to investigate the role of gut microbiota on stress resilience using a rat LH paradigm. First, we investigated whether the composition of the gut microbiota was altered in fecal samples from $\mathrm{LH}$ (susceptible) and non-LH (resilient) rats compared with control rats. Then we examined whether the levels of short-chain fatty acids-acetic acid, propionic acid, butyric acid, lactic acid, and succinic acid-in the fecal samples from susceptible and resilient rats were altered compared with control rats, since these short-chain fatty acids can produced by the gut microbiota ${ }^{23}$.

\section{Materials and methods Animals}

Male Sprague-Dawley rats $(n=25,200-230$ g; 7 weeks, Charles River Japan, Co., Tokyo, Japan) were used. The animals were housed under a 12-h light/dark cycle with ad libitum access to food and water. The experimental procedures were approved by the Chiba University Institutional Animal Care and Use Committee (Permission number: 31-341).

Stress paradigm (LH model) and collection of fecal sample

The LH paradigm was performed as previously reported $^{6-8,11,31-34}$. Animals were initially exposed to uncontrollable stress to create LH rats. When the rats were later placed in a situation in which the shock is controllable; that is, the animal could escape it, an animal exhibiting LH not only fails to acquire an escape response but also often makes no effort to escape the shock at all.

We used the Gemini Avoidance System (San Diego Instruments, San Diego, CA) for LH paradigm. This apparatus has two compartments by a retractable door. On day 1 and day 2, rats were subjected to 30 inescapable electric foot shocks $(0.65 \mathrm{~mA}, 30 \mathrm{~s}$ duration, at random intervals averaging $18-42 \mathrm{~s}$ ). On day 3 , a post-shock test using a two-way conditioned avoidance test was performed to determine whether the rats would exhibit the predicted escape deficits (Fig. 1a). This session consisted of 30 trials, in which electric foot shocks $(0.65 \mathrm{~mA}, 6 \mathrm{~s}$ duration, at random intervals with a mean of $30 \mathrm{~s}$ ) were preceded by a 3-s conditioned stimulus tone that remained on until the shock was terminated. The numbers of escape failures and the latency to escape in each of the 30 trials were counted. Animals with more than 25 escape failures in the 30 trials were regarded as having met the criterion for LH rats (susceptible). Animals with fewer than 24 failures were defined as non-LH rats (resilient $)^{31-34}$. Fresh fecal samples were collected in a blind manner before post-shock stress on day 4, and stored at $-80^{\circ} \mathrm{C}$ until use (Fig. 1A).

\section{S rDNA analysis}

DNA extraction from fecal samples and the 16S rDNA analysis were performed at the TechnoSuruga Laboratory, Co., Ltd. (Shizuoka, Japan), as reported previously ${ }^{35}$. Briefly, the samples were suspended in a buffer containing $4 \mathrm{M}$ guanidium thiocyanate, $100 \mathrm{mM}$ Tris- $\mathrm{HCl}(\mathrm{pH} 9.0)$ and $40 \mathrm{mM}$ EDTA and broken up in the presence of zirconia beads using the FastPrep-24 5G homogenizer (MP Biomedicals, Irvine, CA). Then, DNA was extracted using GENE PREP STAR PI-480 (KURABO, Japan). The final concentration $(10 \mathrm{ng} / \mu \mathrm{L})$ of the DNA sample was used. Briefly, the V3-V4 hypervariable regions of the $16 \mathrm{~S}$ rRNA were amplified from microbial genomic DNA using PCR with the bacterial universal primers $(341 \mathrm{~F} / \mathrm{R} 806)^{35}$ and the dual-index method ${ }^{36}$. For bioinformatics analysis, the overlapping paired-end reads were merged using the fastq-join program with default settings ${ }^{37}$. The reads were processed for quality and chimera filtering as follows. Only reads with quality value scores of 20 for $>99 \%$ of the sequence were extracted, and chimeric sequences were removed using the program usearch6. $1^{38}$. Non-chimeric reads were submitted for $16 \mathrm{~S}$ rDNA-based taxonomic analysis using the Ribosomal Database Project (RDP) Multiclassifier tool ${ }^{39}$. Reads obtained in the Multi-FASTA format were assigned to genus or phylum levels with an $80 \%$ confidence threshold. Principal component analysis (PCA) was performed using Metagenome@KIN software (World Fusion Co., Ltd., Tokyo, Japan) based on data obtained from the bacterial family using the RDP taxonomic analysis software.

\section{Measurement of fecal short-chain fatty acids}

Measurement of short-chain fatty acids-acetic acid, propionic acid, butyric acid, lactic acid, and succinic acid -in fecal samples was performed at the TechnoSuruga Laboratory, Co., Ltd. (Shizuoka, Japan). For the determination of these short-chain fatty acids, feces were suspended in distilled water, heated at $85^{\circ} \mathrm{C}$ for $15 \mathrm{~min}$ to inactivate viruses, and then centrifuged according to previously reported methods ${ }^{40,41}$. The concentrations of these short-chain fatty acids in feces were measured using a high-performance liquid chromatography organic acid analysis system with a Prominence CDD-10A conductivity detector (Shimadzu, Kyoto, Japan), two tandemly arranged Shim-pack SCR-102(H) columns [300 $\mathrm{mm} \times 8 \mathrm{~mm}$ inner diameter (ID)], and a Shim-pack SCR-102(H) guard column $(50 \mathrm{~mm} \times 6 \mathrm{~mm} \mathrm{ID})^{40,41}$. The HPLC calibration curves for the measurement of the short-chain fatty acids were created using prepared standard solutions. 


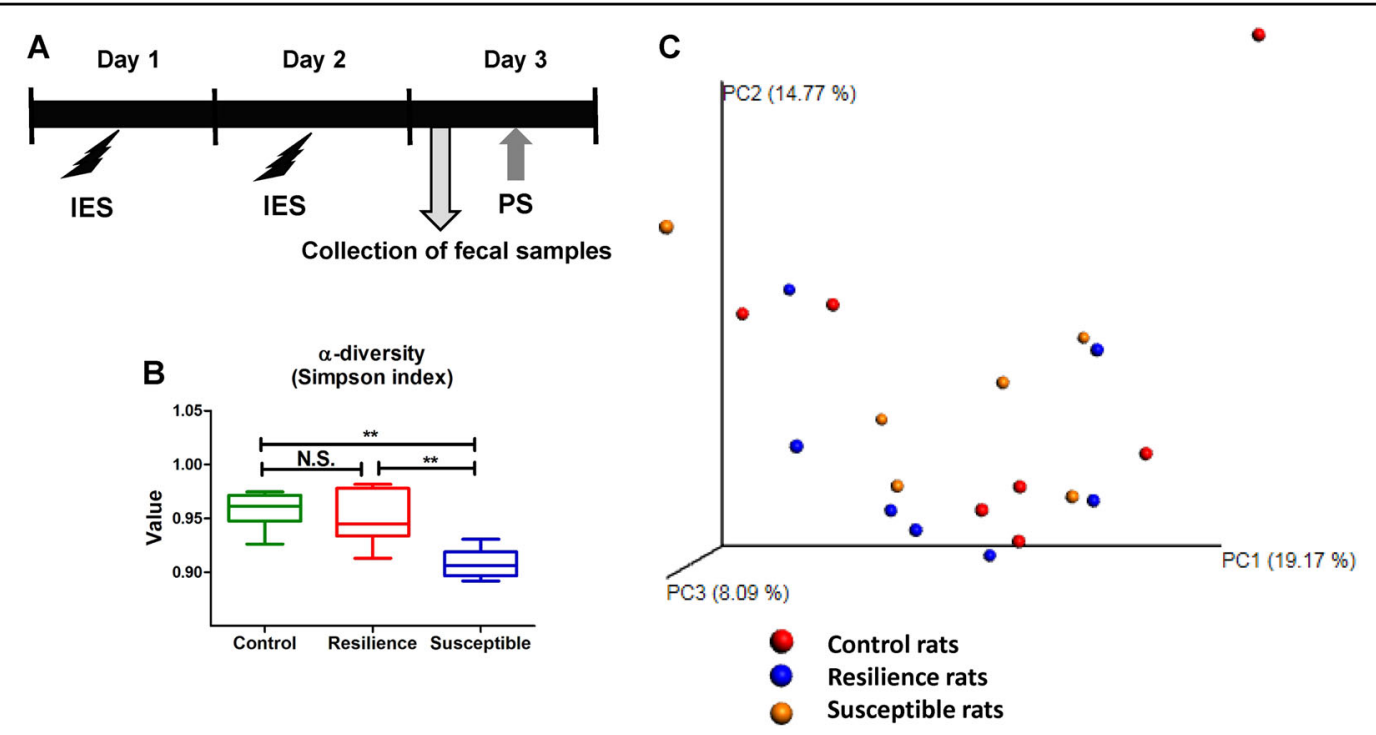

Fig. 1 Experimental schedule of LH paradigm and profiles of gut microbiota. a Schedule of LH paradigm and collection of fecal samples. Rats received inescapable electric shock (IES) treatments on 2 days (days 1 and 2). On day 3, fecal samples from rats were collected. Subsequently, rats passed a post-shock test (PS), and were designated as resilient rats and susceptible rats. b Simpson index (an a-diversity indicator: one-way ANOVA: $\left.F_{2,17}=11.531, P=0.001\right)$ among the three groups. a-Diversity data are shown as mean \pm S.E.M. $(n=6$ or 7$) .{ }^{*} P<0.01$. N.S.: not significant. c Principal coordinates analysis (PCA)

\section{Statistical analysis}

The data are presented as the mean \pm standard error of the mean (S.E.M.). Analysis was performed using the PASW Statistics 20 software (now SPSS statistics; SPSS, Tokyo, Japan). Comparisons between groups were performed using one-way analysis of variance, followed by post hoc Fisher's Least Significant Difference test. A $P$ value $<0.05$ was considered statistically significant.

\section{Results}

\section{Composition of gut microbiota in control, resilient, and} susceptible rats

We used 16S rDNA gene sequencing to determine differences in the gut microbiota composition among the three groups of rats. $\alpha$-diversity refers to the diversity of bacteria or species within a community or habitat. The susceptible rats showed a significant decrease in the $\alpha$ diversity value compared with control rats or resilient rats (Fig. 1b). In the three-dimensional PCoA data, the measurements from susceptible rats were well separated from those of control rats and resilient rats. Four measurements from the susceptible group were close to those of the sham group, whereas the other three measurements were close to those of the resilient group (Fig. 1c).

Firmicutes were the most dominant phylum, comprising $>85 \%$ of the total sequences. There were no significant differences in the levels of this phylum among the three groups. The order levels of gut bacterium in control rats, resilient rats, and susceptible rats were identified (Fig. 2a).
Clostridiales and Lactobacillales were the most dominant orders, with $>80 \%$ of total sequences. The number of Lactobacillales was significantly increased in the susceptible rats compared with that in control and resilient rats (Fig. 2b). In contrast, the number of Lactobacillales in the resilient rats was similar to that in the control rats (Fig. 2b). The number of Actinomycetales in the susceptible rats was significantly lower than that in the resilient rats (Fig. 2c). Although the number of Actinomycetales in the resilient rats was higher than that in the control rats, the difference did not reach statistical significance.

The families of the gut bacteria in control, resilient, and susceptible rats are shown (Fig. 3a). Lactobacillaceae were significantly more highly represented in the susceptible rats than in the control and resilient rats, although the number of Lactobacillaceae in the resilient rats was similar to that in the control rats (Fig. 3b). In contrast, the number of Corynebacteriaceae was significantly lower in the susceptible rats than that in the resilient rats, although these two did not significantly differ from the control rats (Fig. 3c).

The genera of gut bacteria in the control, resilient, and susceptible rats are shown (Fig. 4a). Lactobacillus, Clostridium cluster III, and Anaerofustis numbers were significantly higher in the susceptible rats than in the control and resilient rats (Fig. 4b, c, e). Corynebacterium numbers were significantly lower in the susceptible rats than in the resilient rats, although these two groups were not significantly altered compared with the control rats (Fig. 4d). 


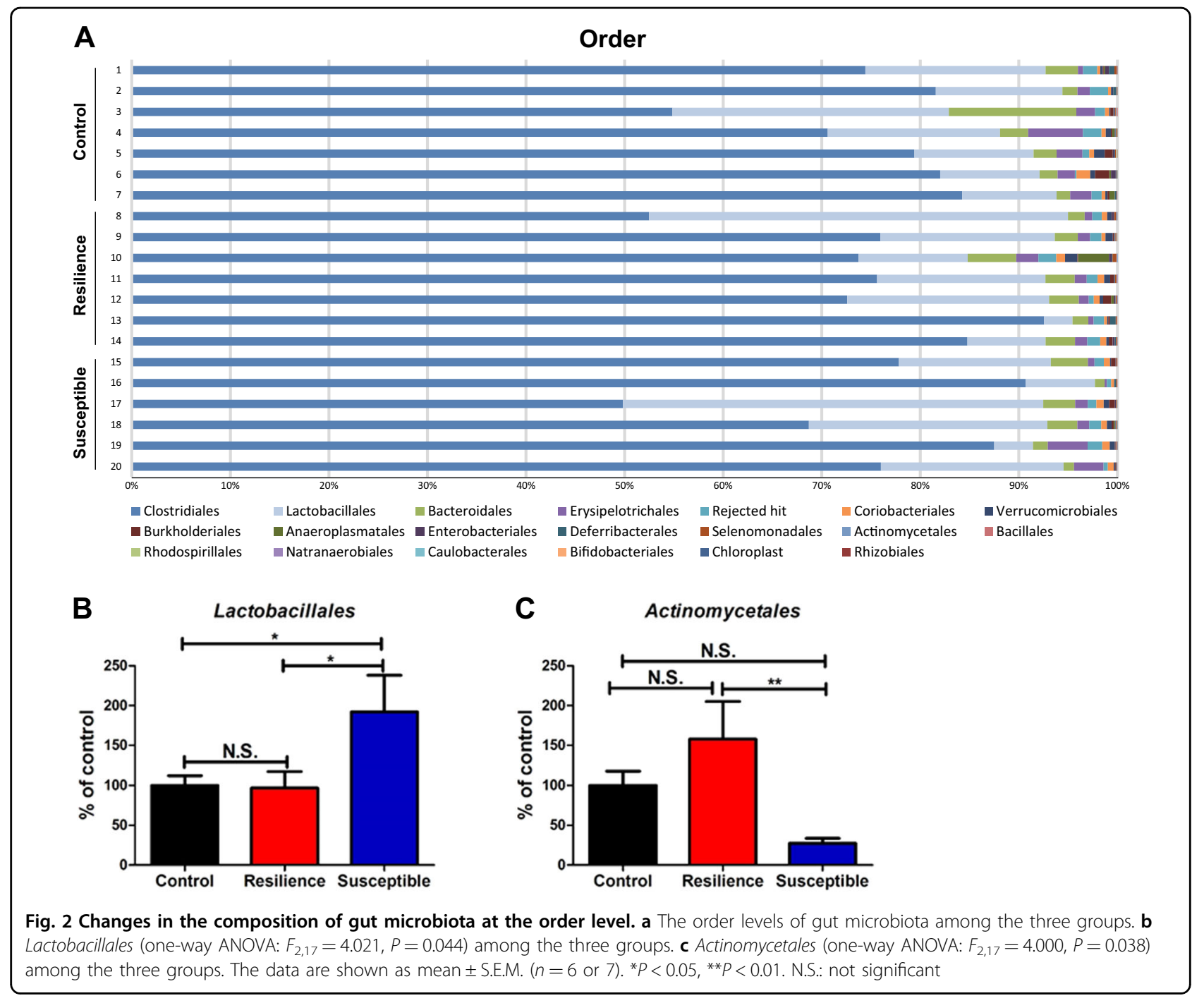

\section{Measurement of short-chain fatty acids in fecal samples}

Levels of acetic acid and propionic acid in the susceptible rats were significantly lower than those in control rats and resilient rats, and there were no significant differences between control rats and resilient rats (Fig. 5a, b). There were no changes in butyric acid and succinic acid among the three groups (Fig. 5c, e). In contrast, levels of lactic acid in the susceptible rats were significantly higher than those of control rats and resilient rats, although there were no changes between control rats and resilient rats (Fig. $5 d)$. There was a positive correlation $(r=0.461, P=$ 0.041 ) between lactic acid and Lactobacillus levels among three groups (Fig. 5f). There were no correlations between other short-chain fatty acids and the microbiome composition among the three experimental groups.

\section{Discussion}

The major findings of this study are as follows. At the order level, susceptibility to LH in rats exposed to inescapable shock might be associated with an increase of Lactobacillales and a decrease of Actinomycetales in the host gut. At the family level, stress susceptibility might be associated with an increase in Lactobacillaceae and decrease of Corynebacteriaceae in the host gut. At the gene level, stress susceptibility might be associated with the increase of Lactobacillus, Clostridium cluster III and Anaerofustis, and the decrease of Corynebacterium in the host gut. Levels of acetic acid and propionic acid in the feces from susceptible rats were lower than those in the feces of control and resilient rats, whereas levels of lactic acid in the susceptible rats were higher than those of control and resilient rats. There was a positive correlation between lactic acid and Lactobacillus levels among these three groups. These findings suggest that alterations in the composition of these microbiota contribute to susceptibility versus resilience in rats in the LH situation.

In this study, we found an increase in the abundance of members of the order Lactobacillales and the family 


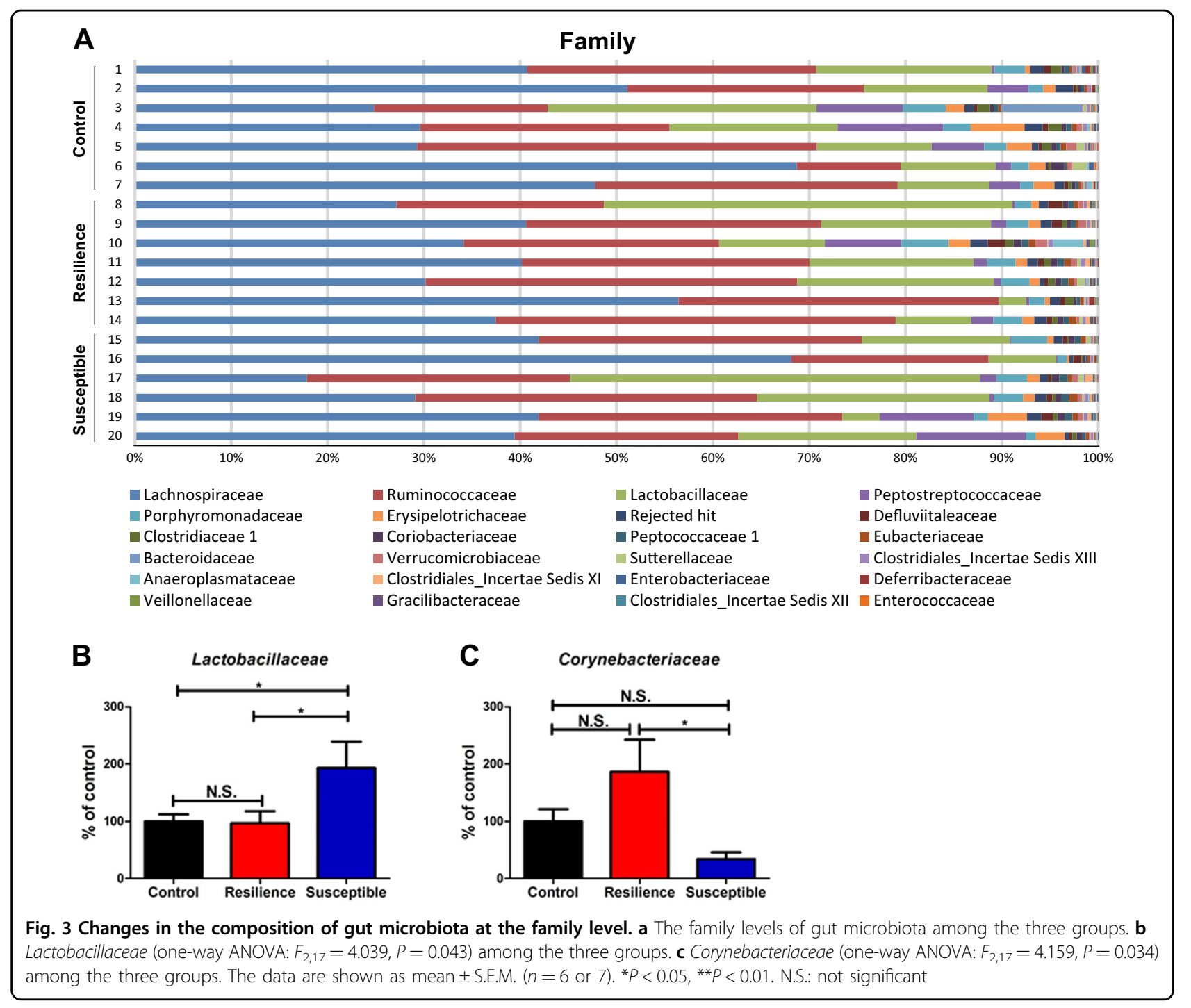

Lactobacillaceae in susceptible rats, in comparison with control and resilient rats. It is possible that increased abundance of members of the Lactobacillales order and Lactobacillaceae family might play a role in susceptibility versus resilience of rats to $\mathrm{LH}$ after inescapable electric stress.

At the genus level, susceptibility in rats exposed to inescapable shock might be associated with an increase in Lactobacillus, Clostridium cluster III, and Anaerofustis and a decrease of Corynebacterium in the host gut. Clostridium, a genus of Gram-positive bacteria, includes several significant human pathogens, such as the causative agent of botulism. High levels of members of the genus Clostridium have been reported in patients with major depressive disorder (MDD) compared with controls ${ }^{42,43}$, suggesting that increased levels of Clostridium play a role in depression. We reported that susceptible mice after CSDS have higher levels of Clostridium, and that the novel antidepressant candidate $(R)$-ketamine attenuated the increased levels of Clostridium in susceptible mice ${ }^{25}$. This study shows that the antidepressant effects of $(R)$ ketamine might be partly mediated by the restoration of altered composition of the gut microbiota in the CSDS susceptible mice. Although the role of members of Clostridium cluster III in depression is currently unclear, it appears that Clostridium cluster III may contribute to susceptibility in rats subjected to inescapable electric stress.

Anaerofustis is a strictly anaerobic, Gram-positive, rodshaped, non-spore-forming bacterial genus of the family Eubacteriaceae $^{44}$. In this study, we found decreased levels of Anaerofustis in the susceptible rats compared with the control and resilient rats. At present, there have been no reports showing alterations in Anaerofustis in patients with MDD, or in rodents with depression-like phenotypes. Therefore, it is unclear how decreased levels of 


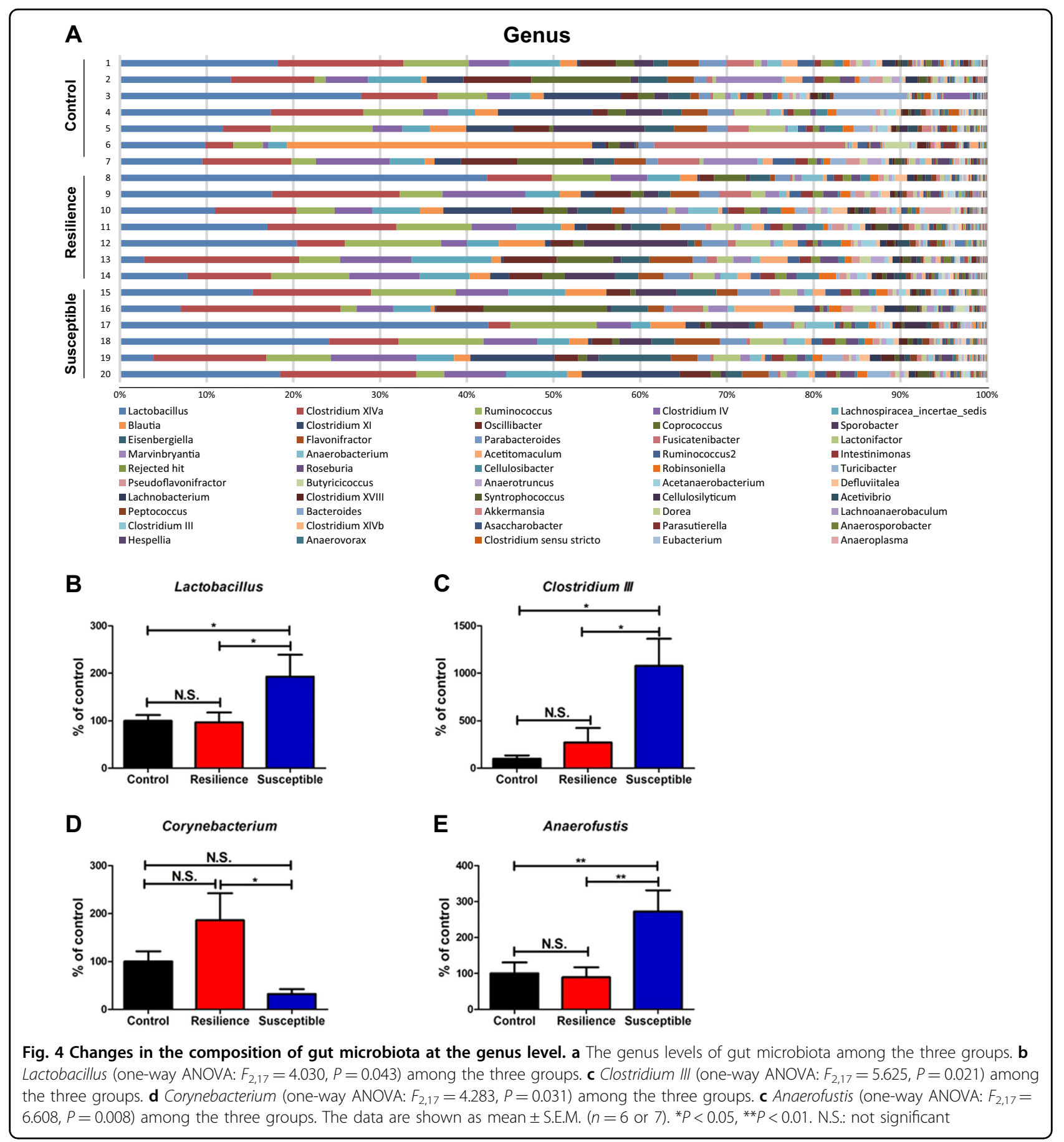

Anaerofustis play a role in susceptibility to LH. Further study into the role of Anaerofustis in depression is needed.

In this study, we found lower levels of Corynebacterium in the susceptible rats compared with control and resilient rats. It has been reported that sub-chronic and chronic exposure to glyphosate-based herbicides decreases the composition of microbiota, such as Corynebacterium, resulting in behavioral abnormalities including depression and anxiety ${ }^{45}$. Low levels of Corynebacterium were also reported in a chronic variable stress-induced rat model of depression $^{46}$. It appears likely that lowered levels of Corynebacterium might play a role in a depression-like phenotype in rodents, although further study into the role of Corynebacterium in depression is needed.

Short-chain fatty acids-acetic acid, propionic acid, butyric acid, lactic acid, and succinic acid-are generated as the end products of the degradation and fermentation of indigestible carbohydrates by the gut microbiota ${ }^{47}$. 

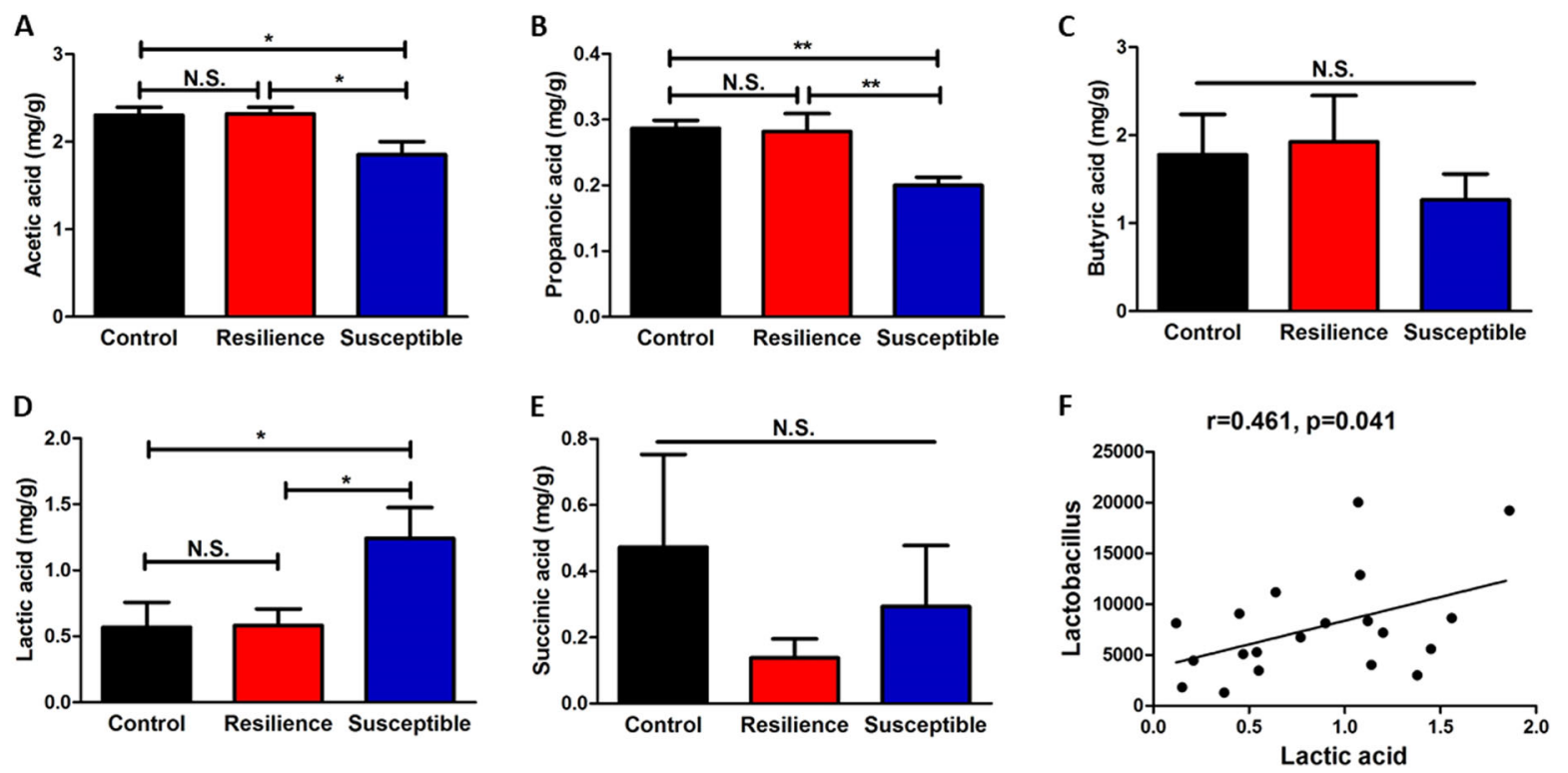

Fig. 5 Levels of short-chain fatty acids in fecal samples and correlation with microbiota. a Acetic acid (one-way ANOVA: $F_{2,17}=5.898, P=$ 0.016 ) among the three groups. b Propionic acid (one-way ANOVA: $F_{2,17}=8.175, P=0.004$ ) among the three groups. $\mathbf{c}$ Butyric acid (one-way ANOVA: $F_{2,17}=0.559, P=0.582$ ) among the three groups. $\mathbf{d}$ Lactic acid (one-way ANOVA: $F_{2,17}=4.219, P=0.041$ ) among the three groups. e Succinic acid (one-way ANOVA: $\left.F_{2,17}=0.763, P=0.488\right)$ among the three groups. The data are shown as mean \pm S.E.M. $(n=6$ or 7$)$. ${ }^{*} P<0.05$, **P $<0.01$. N.S.: not significant. $\mathbf{f}$ There is a positive correlation $(r=0.461, P=0.041)$ between lactic acid and Lactobacillus in fecal samples

Measurement of these short-chain fatty acids could therefore serve as an indirect method for the analysis of microbiota composition. These organic acids have specific anti-microbial activities ${ }^{48}$. It has been reported that levels of acetic acid and propionic acid in feces from women with depression are lower than those in control subjects, and that there are negative correlations between acetic acid or propionic acid and depression scores ${ }^{49}$. In this study, we found decreased levels of acetic acid and propionic acid in rats susceptible to $\mathrm{LH}$, compared with control and resilient rats, consistent with the results from a recent clinical study ${ }^{49}$. We also found decreased levels of butyric acid in the susceptible rats, although the difference did not reach statistical significance. Three shortchain fatty acids containing $\mathrm{C} 2-\mathrm{C} 4$, acetic acid, propionic acid, and butyric acid, account for over $95 \%$ of the pool of short-chain fatty acids ${ }^{23}$. It is likely that decreased levels of acetic acid and propionic acid in feces may be associated with susceptibility to LH, and with depression in patients with MDD.

Elevated levels of lactic acid in the blood, cerebrospinal fluid and brain have been reported in patients with $\mathrm{MDD}^{50-52}$. Higher levels of lactic acid in rodents with depression-like behaviors have also been reported ${ }^{53}$. In contrast, peripheral administration of lactic acid produced antidepressant-like effects in different models of depres$\operatorname{sion}^{54}$. In this research we found higher levels of lactic acid in feces from susceptible rats compared with those from control and resilient rats. We found a positive correlation between Lactobacillus, microbes which produce lactic acid, and the amounts of lactic acid in fecal samples. It appears likely that the increased levels of lactic acid produced by Lactobacillus might contribute to susceptibility to inescapable electric stress, although further study is needed.

The crosstalk between the brain and the gut is predominately influenced by the gut bacteria ${ }^{55}$. Imbalance of gut microbiota has been found to cause abnormalities in the brain-gut axis in several neurological and psychiatric diseases $^{13,55}$. Multiple lines of evidence suggest that an abnormal composition of the gut microbiota contributes to the resilience or susceptibility to LH in rodents after repeated stress ${ }^{30,56-59}$. It is well recognized that gut microbiota plays a role in animal behaviors ${ }^{14,60-62}$, although the precise mechanisms underlying the microbiome-mediated behaviors are currently unknown. For example, it has been reported that the vagus nerve plays a major role in modulating the constitutive communication pathway between the brain and the bacteria in the gut $^{63,64}$. It is likely that altered composition of microbiota might play a key role in the stress-induced disorders although further study is needed.

This research has some limitations. In this study, we did not identify the specific microbiome which can affect susceptibility or resilience in the LH experiments. Therefore, from the present data, we do not know how the specific microbiome can affect behaviors under the LH paradigm. In the future, it will be necessary to identify 
the specific microbiome, using approaches such as shotgun metagenomics sequencing. It will also be of interest to investigate the way in which specific microbiomes affect behaviors related to $\mathrm{LH}$.

In conclusion, the present study suggests that an altered composition of the gut microbiota, including organisms, such as Lactobacillus, Clostridium cluster III, Anaerofustis, and Corynebacterium, contributes to resilience and susceptibility to learned helplessness in rats subjected to inescapable electric foot shock.

\section{Acknowledgements}

This study was supported by SENSHIN Medical Research Foundation, Japan (to K.Z.), Smoking Research Foundation, Japan (to K.H.), and AMED, Japan (to K.H., JP19dm0107119). L.C. was supported by Japan China Sasakawa Medical Fellowship (Tokyo, Japan). S.W. was supported by TAKASE Scholarship Foundation (Tokyo, Japan).

\section{Author details}

'Division of Clinical Neuroscience, Chiba University Center for Forensic Mental Health, Chiba 260-8670, Japan. ${ }^{2}$ Department of Psychiatry, Chaohu Hospital of Anhui Medical University, 238000 Hefei, China. ${ }^{3}$ Department of Psychiatry, Teikyo University Chiba Medical Center, Ichihara, Chiba 299-0111, Japan

\section{Conflict of interest}

K.H. has received research support from Dainippon-Sumitomo, Otsuka, and Taisho. The remaining authors declare that they have no conflict of interest.

\section{Publisher's note}

Springer Nature remains neutral with regard to jurisdictional claims in published maps and institutional affiliations.

Received: 10 May 2019 Revised: 29 June 2019 Accepted: 30 July 2019 Published online: 17 September 2019

\section{References}

1. Southwick, S. M., Vythilingam, M. \& Charney, D. S. The psychobiology of depression and resilience to stress: implications for prevention and treatment. Ann. Rev. Clin. Psychol. 1, 255-291 (2005).

2. Feder, A., Nestler, E. J. \& Charney, D. S. Psychobiology and molecular genetics of resilience. Nat. Rev. Neurosci. 10, 446-457 (2009).

3. Franklin, T. B., Saab, B. J. \& Mansuy, I. M. Neural mechanisms of stress resilience and vulnerability. Neuron 75, 747-761 (2012).

4. Russo, S. J., Murrough, J. W., Han, M. H., Charney, D. S. \& Nestler, E. J. Neurobiology of resilience. Nat. Neurosci. 15, 1475-1484 (2012).

5. Taliaz, D. et al. Resilience to chronic stress is mediated by hippocampal brainderived neurotrophic factor. J. Neurosci. 31, 4475-4483 (2012).

6. Yang, C., Shirayama, Y., Zhang, J. C., Ren, Q. \& Hashimoto, K. Regional differences in brain-derived neurotrophic factor levels and dendritic spine density confer resilience to inescapable stress. Int. J. Neuropsychopharmacol. 18, pyu121 (2015).

7. Yang, C., Shirayama, Y., Zhang, J. C., Ren, Q. \& Hashimoto, K. Peripheral interleukin-6 promotes resilience versus susceptibility to inescapable electric stress. Acta Neuropsychiatr. 27, 312-316 (2015).

8. Yang, B. et al. Regional differences in the expression of brain-derived neurotrophic factor (BDNF) pro-peptide, proBDNF and preproBDNF in the brain confer stress resilience. Eur. Arch. Psychiatry Clin. Neurosci. 266, 765-769 (2016)

9. Dantzer, R., Cohen, S., Russo, S. J. \& Dinan, T. G. Resilience and immunity. Brain Behav. Immun. 74, 28-42 (2018).

10. Qu, Y. et al. Regional differences in dendritic spine density confer resilience to chronic social defeat stress. Acta Neuropsychiatr. 30, 117-122 (2018).
11. Zhang, J. C. et al. Keap1-Nrf2 signaling pathway confers resilience versus susceptibility to inescapable electric stress. Eur. Arch. Psychiatry Clin. Neurosci. 268, 865-870 (2018).

12. Dinan, T. G. \& Cryan, J. F. Brain-gut-microbiota axis and mental health. Psychosom. Med. 79, 920-926 (2017).

13. Fung, T. C., Olson, C. A. \& Hsiao, E. Y. Interactions between the microbiota, immune and nervous systems in health and disease. Nat. Neurosci. 20, 145-155 (2017).

14. Cussotto, S., Sandhu, K. V., Dinan, T. G. \& Cryan, J. F. The neuroendocrinology of the microbiota-gut-brain axis: a behavioural perspective. Front. Neuroendocrinol. 51, 80-101 (2018).

15. Molina-Torres, G., Rodriguez-Arrastia, M., Roman, P., Sanchez-Labraca, N. \& Cardona, D. Stress and the gut microbiota-brain axis. Behav. Pharmacol. 30(2 and 3 Special Issue), 187-202 (2019).

16. Cussotto $\mathrm{S}$., et al. Differential effects of psychotropic drugs on microbiome composition and gastrointestinal function. Psychopharmacology (Berl.). https:// doi.org/10.1007/s00213-018-5006-5 (2018).

17. Jiang, $\mathrm{H}$. et al. Altered fecal microbiota composition in patients with major depressive disorder. Brain Behav. Immun. 48, 186-194 (2015).

18. Zheng, P. et al. Gut microbiome remodeling induces depressive-like behaviors through a pathway mediated by the host's metabolism. Mol. Psychiatry 21, 786-796 (2016).

19. Wong, M. L. et al. Inflammasome signaling affects anxiety- and depressive-like behavior and gut microbiome composition. Mol. Psychiatry 21, 797-805 (2016).

20. Huang, T. T. et al. Current understanding of gut microbiota in mood disorders: an update of human studies. Front. Genet. 10, 98 (2019).

21. Yang, C. et al. Key role of gut microbiota in anhedonia-like phenotype in rodents with neuropathic pain. Transl. Psychiatry 9, 57 (2019).

22. Burokas, A. et al. Targeting the microbiota-gut-brain axis: prebiotics have anxiolytic and antidepressant-like effects and reverse the impact of chronic stress in mice. Biol. Psychiatry 82, 472-487 (2017).

23. den Besten, G. et al. The role of short-chain fatty acids in the interplay between diet, gut microbiota, and host energy metabolism. J. Lipid Res. 54, 2325-2340 (2013)

24. Ho, P. \& Ross, D. A. More than a gut feeling: the implications of the gut microbiota in psychiatry. Biol. Psychiatry 81, e35-e37 (2017).

25. $\mathrm{Qu}$, Y. et al. Comparison of (R)-ketamine and lanicemine on depression-like phenotype and abnormal composition of gut microbiota in a social defeat stress model. Sci. Rep. 7, 15725 (2017).

26. Yang, C. et al. Possible role of the gut microbiota-brain axis in the antidepressant effects of (R)-ketamine in a social defeat stress model. Transl. Psychiatry 7, 1294 (2017).

27. Zhang, J. C. et al. Blockade of interleukin-6 receptor in the periphery promotes rapid and sustained antidepressant actions: a possible role of gut-microbiotabrain axis. Transl. Psychiatry 7, e1138 (2017).

28. Huang, N. et al. Role of Actinobacteria and Coriobacteriia in the antidepressant effects of ketamine in an inflammation model of depression. Pharmacol. Biochem. Behav. 176, 93-100 (2019).

29. Lukić, I. et al. Antidepressants affect gut microbiota and Ruminococcus flavefaciens is able to abolish their effects on depressive-like behavior. Transl. Psychiatry 9, 133 (2019).

30. Yang, C. et al. Bifidobacterium in the gut microbiota confer resilience to chronic social defeat stress in mice. Sci. Rep. 7, 45942 (2017).

31. Muneoka, K, Shirayama, Y., Horio, M., Iyo, M. \& Hashimoto, K. Differential levels of brain amino acids in rat models presenting learned helplessness or nonlearned helplessness. Psychopharmacol. (Berl.) 226, 63-71 (2013).

32. Shirayama, Y. et al. Alterations in brain-derived neurotrophic factor (BDNF) and its precursor proBDNF in the brain regions of a learned helplessness rat model and the antidepressant effects of a TrkB agonist and antagonist. Eur. Neuropsychopharmacol. 25, 2449-2458 (2015).

33. Shirayama, Y. \& Hashimoto, K. Effects of a single bilateral infusion of R-ketamine in the rat brain regions of a learned helplessness model of depression. Eur. Arch. Psychiatry Clin. Neurosci. 267, 177-182 (2017).

34. Shirayama, Y. \& Hashimoto, K. Lack of antidepressant effects of $(2 R, 6 R)$ hydroxynorketamine in a rat learned helplessness model: Comparison with (R)-ketamine. Int. J. Neuropsychopharmacol. 21, 84-88 (2018).

35. Takahashi, S., Tomita, J., Nishioka, K., Hisada, T. \& Nishijima, M. Development of a prokaryotic universal primer for simultaneous analysis of Bacteria and Archaea using next-generation sequencing. PLoS ONE 9, e105592 (2014). 
36. Hisada, T., Endoh, K. \& Kuriki, K. Inter- and intra-individual variations in seasonal and daily stabilities of the human gut microbiota in Japanese. Arch. Microbiol. 197, 919-934 (2015).

37. Erik A. ea-utils "Command-line tools for processing biological sequencing data". http://code.google.com/p/ea-utils (2011).

38. Edgar, R. C., Haas, B. J., Clemente, J. C., Quince, C. \& Knight, R. UCHIME improves sensitivity and speed of chimera detection. Bioinformatics 27, 2194-2200 (2011).

39. Wang, Q., Garrity, G. M., Tiedje, J. M. \& Cole, J. R. Naïve Bayesian classifier for rapid assignment of rRNA sequences into the new bacterial taxonomy. Appl. Environ. Microbiol. 72, 5261-5267 (2007)

40. Higashimura, Y. et al. Protective effect of agaro-oligosaccharides on gut dysbiosis and colon tumorigenesis in high-fat diet-fed mice. Am. J. Physiol. Gastrointest. Liver Physiol. 310, G367-G375 (2016).

41. Aoe, S., Nakamura, F. \& Fujiwara, S. Effect of wheat bran on fecal butyrateproducing bacteria and wheat bran combined with barley on Bacteroides abundance in Japanese healthy adults. Nutrients 10, 1980 (2018).

42. Lin, P. et al. Prevotella and Klebsiella proportions in fecal microbial communities are potential characteristic parameters for patients with major depressive disorder. J. Affect. Disord. 207, 300-304 (2017).

43. Rong, $\mathrm{H}$. et al. Similarly in depression, nuances of gut microbiota: Evidences from a shotgun metagenomics sequencing study on major depressive disorder versus bipolar disorder with current major depressive episode patients. J. Psychiatr. Res. 113, 90-99 (2019).

44. Finegold, S. M. et al. Anaerofustis stercorihominis gen. nov., sp. nov., from human feces. Anaerobe 10, 41-45 (2004).

45. Aitbali, Y. et al. Glyphosate based- herbicide exposure affects gut microbiota, anxiety and depression-like behaviors in mice. Neurotoxicol. Teratol. 67, 44-49 (2018).

46. Yu, M. et al. Variations in gut microbiota and fecal metabolic phenotype associated with depression by $16 \mathrm{~S}$ rRNA gene sequencing and LC/MS-based metabolomics. J. Pharm. Biomed. Anal. 138, 231-239 (2017).

47. Koh, A., De Vadder, F., Kovatcheva-Datchary, P. \& Backhed, F. From dietary fiber to host physiology: short-chain fatty acids as key bacterial metabolites. Cell 165, 1332-1345 (2016).

48. Dibner, J. J. \& Buttin, P. Use of organic acids as a model to study the impact of gut microflora on nutrition and metabolism. J. Appl. Poult. Res. 11, 453-463 (2002).

49. Skonieczna-Żydecka, K. et al. Faecal short chain fatty acids profile is changed in Polish depressive women. Nutrients 10, E1939 (2018).
50. Levine, J., Panchalingam, K., McClure, R. J., Gershon, S. \& Pettegrew, J. W. Stability of CSF metabolites measured by proton NMR. J. Neural Transm. Vienna). 107, 843-848 (2000).

51. Regenold, W. T. et al. Elevated cerebrospinal fluid lactate concentrations in patients with bipolar disorder and schizophrenia: implications for the mitochondrial dysfunction hypothesis. Biol. Psychiatry 65, 489-494 (2009).

52. Ernst, J. et al. Increased pregenual anterior cingulate glucose and lactate concentrations in major depressive disorder. Mol. Psychiatry 22, 113-119 (2017)

53. Shi, B. et al. A ${ }^{1} \mathrm{H}-\mathrm{NMR}$ plasma metabonomic study of acute and chronic stress models of depression in rats. Behav. Brain. Res. 241, 86-91 (2013).

54. Carrard, A. et al. Peripheral administration of lactate produces antidepressantlike effects. Mol. Psychiatry 23, 392-399 (2018).

55. Kelly, J. R., Clarke, G., Cryan, J. F. \& Dinan, T. G. Brain-gut-microbiota axis: challenges for translation in psychiatry. Ann. Epidemiol. 26, 366-372 (2016).

56. Szyszkowicz, J. K., Wong, A., Anisman, H., Merali, Z. \& Audet, M. C. Implications of the gut microbiota in vulnerability to the social avoidance effects of chronic social defeat in male mice. Brain Behav. Immun. 66, 45-55 (2017).

57. Hao, Z., Wang, W., Guo, R. \& Liu, H. Faecalibacterium prausnitzii (ATCC 27766) has preventive and therapeutic effects on chronic unpredictable mild stressinduced depression-like and anxiety-like behavior in rats. Psychoneuroendocrinology 104, 132-142 (2019).

58. Kentner, A. C., Cryan, J. F. \& Brummelte, S. Resilience priming: Translationa models for understanding resiliency and adaptation to early life adversity. Dev. Psychobiol. 61, 350-375 (2019).

59. Gururajan, A. et al. Resilience to chronic stress is associated with specific neurobiological, neuroendocrine and immune responses. Brain Behav. Immun. https://doi.org/10.1016/j.bbi.2019.05.004 (2019).

60. Ezewa, V. O., Gerardo, N. M., Inouye, D. W., Medina, M. \& Xavier, J. B. Animal behavior and microbiome. Science 338, 198-199 (2012).

61. Borre, Y. E., Moloney, R. D., Clarke, G., Dinan, T. G. \& Cryan, J. F. The impact of microbiota on brain and behavior: mechanisms \& therapeutic potential. Adv Exp. Med. Biol. 817, 373-403 (2014).

62. Jianguo, L., Xueyang, J., Cui, W., Changxin, W. \& Xuemei, Q. Altered gut metabolome contributes to depression-like behaviors in rats exposed to chronic unpredictable mild stress. Transl. Psychiatry 9, 40 (2019).

63. Bravo, J. A. et al. Ingestion of Lactobacillus strain regulates emotional behavior and central GABA receptor expression in a mouse via the vagus nerve. Proc. Natl Acad. Sci. USA 108, 16050-16055 (2011).

64. Dinan, T. G. \& Cryan, J. F. Gut microbiomes and depression: still waiting for godot. Brain Behav. Immun. 79, 1-2 (2019). 